\title{
Absolute motion parallax and the specific distance tendency*
}

\author{
WALTER C. GOGEL and JEROME D. TIETZ \\ University of California. Santa Barbara. California 93106
}

\begin{abstract}
In the absence of definitive cues to distance, the perceived distance of an object will be in error in the direction of the object appearing at a distance of about $2 \mathrm{~m}$ from 0 . This tendency to perceive an object at a relatively near distance is termed the specific distance tendency (Gogel. 1969). Also. it has been found that an error in perceiving the distance of an object will result in an apparent movement of the object when the head is moved (Hay \& Sawyer. 1969: Wallach. Yablick. \& Smith. 1972). From these two results, it was expected that the direction of the apparent movement of a stationary point of light resulting from head movement would vary predictably as a function of the physical distance of the point of light from $O$. This expectation was confirmed in an experiment in which both the perceived motion and perceived distance of the point of light were measured. The consequences of the study for the role of motion parallax in the perception of distance and for the reafference principle in the perception of object motion with head motion are discussed.
\end{abstract}

A physically stationary object can appear stationary despite changes in the direction of the object with respect to the $O$ as a consequence of head motion (Wallach \& Kravitz, 1965a). It has been found, however, that if cues to the distance of a point of light from $O$ are varied with the change in the egocentric direction of the light resulting from head motion held constant, the physically stationary light will appear to move (Hay \& Sawyer, 1969; Wallach, Yablick, \& Smith, 1972). For a constant amount of head movement, the change in the egocentric direction of a point of light is specified by the distance of the light from 0 . These studies can be interpreted, therefore, as indicating that the perception of motion of a physically stationary light as a function of head motion is related to the difference between the physical distance, $D$, and perceived distance, $D^{\prime}$, of the light.

In the above studies, $\mathrm{D}-\mathrm{D}^{\prime}$ was varied by modifying either the convergence (Hay \& Sawyer, 1969) or both the convergence and accommodation (Wallach, Yablick, \& Smith, 1972) to the light for a particular value of $D$. In the present experiment, the effect of $D-D^{\prime}$ will be studied by presenting a point of light at different distances from $O$ with cues of distance severely reduced. It has been found under these conditions that an object will tend to be perceived at a relatively near distance from $O$ (about $2 \mathrm{~m}$ ) regardless of its physical distance. This has been termed the specific distance tendency (Gogel, 1969). Under these conditions, positioning the point of light farther than the distance defined by the specific distance tendency (SDT) should produce a positive $D-D^{\prime}$, and positioning the light nearer than the SDT should result in a negative $D-D^{\prime}{ }^{1}$ Only at the distance defined by the SDT should D - D' equal zero.

*This investigation was supported by PHS Research Grant NS 08883 from the National Institute of Neurological Diseases and Stroke. The authors wish to thank Robert $E$. Newton for his help in the collection and analysis of the data.
As will be indicated below, the perceived direction of motion of the stationary light as a result of head motion should be in the same direction as the head motion when $D-D^{\prime}$ is positive and opposite to the direction of the head motion when $D-D^{\prime}$ is negative. Only when the light is at the distance of the SDT should the light appear stationary with head motion. The main purpose of this study is to test the validity of the SDT by determining whether at some relatively near distance from $O$ the direction of the apparent motion of the physically stationary light will change with respect to the direction of the head motion, using relatively reduced conditions of observation.

The expected effect of $D-D^{\prime}$ upon the perceived motion of a stationary light as a result of head motion can be considered with the aid of Fig. 1. The upper drawing of Fig. 1 illustrates the physical situation in which a stationary point of light, $f$, is physically at a distance $D_{f}$ from $O$. As $O$ moves his head laterally from Position 1 to Position 2, the physical direction of the point of light with respect to $O$ changes from $\phi_{1}$ to $\phi_{2}$ through an angle $\phi_{\mathrm{T}}$, where $\phi_{\mathrm{T}}=\phi_{1}+\phi_{2}$.

The sensed or apparent characteristics are indicated by the lower drawing and the prime notation. Three possible apparent distances of the point of light from $O$ are considered. If the point of light appears at the distance $D_{e}^{\prime}$, the point of light will appear to move a distance $m_{e}^{\prime}$ (from $e_{1}^{\prime}$ to $e_{2}^{\prime}$ ) as the head is moved from Position 1 to Position 2, with this apparent motion in the same direction as that of the head motion. If the point of light appears at the distance $D_{f}^{\prime}$, the point of light will appear to be stationary $\left(f_{1}^{\prime}=f_{2}^{\prime}\right)$ as the head is moved. If the point of light appears at the distance $D_{g}^{\prime}$, the point of light will appear to move a distance $\mathrm{m}_{\mathrm{g}}^{\prime}$ (from $g_{1}^{\prime}$ to $g_{2}^{\prime}$ ) as the head is moved from Position 1 to Position 2, with this apparent motion opposite in direction to that of the head motion. Thus, if the apparent distance of the point of light is less than, equal to, or greater than $D_{f}^{\prime}$, it will appear to move in the 

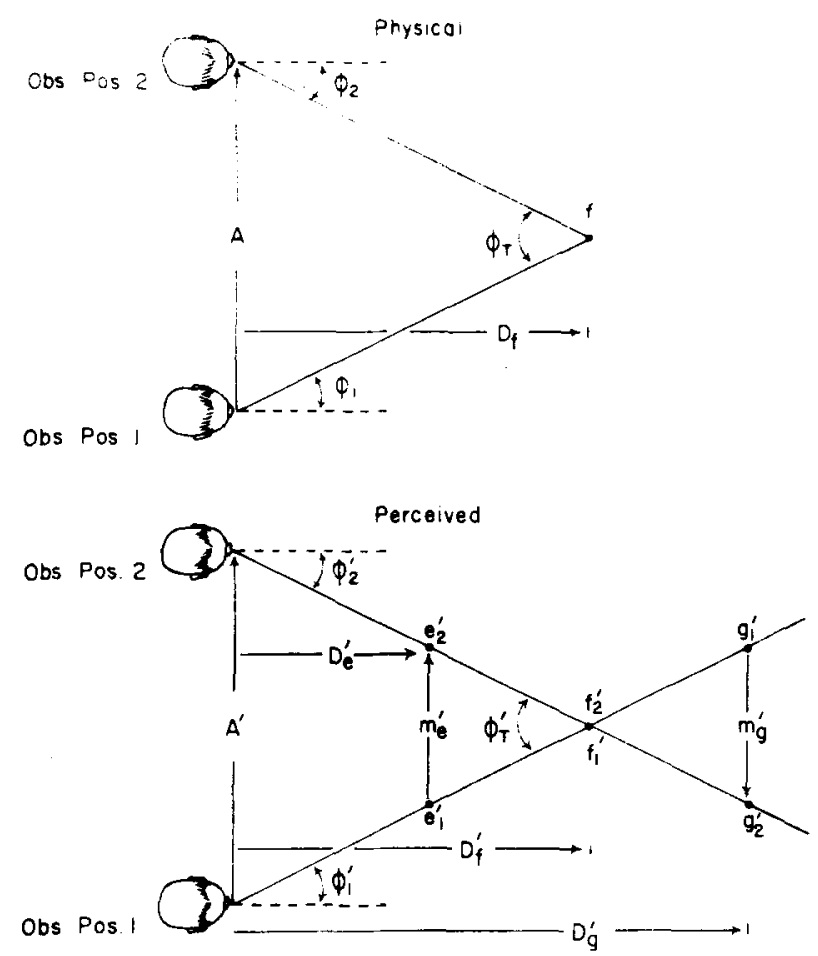

Fig. 1. Schematic top-view drawing for considering the physical and perceptual variables important in the perception of the motion of a stationary point with moving head.

direction of the head motion, appear stationary, or will appear to move opposite to the direction of the head motion, respectively.

For values of $\phi_{\mathrm{T}}^{\prime}$ for which $\phi_{\mathbf{T}}^{\prime}$ in radians and $\tan \phi_{\mathrm{T}}^{\prime}$ can be considered as equal, it follows that

$$
m^{\prime}=\phi_{T}^{\prime}\left(\bar{D}_{f}^{\prime}-D_{n}^{\prime}\right)=\frac{A^{\prime}\left(D_{f}^{\prime}-D_{n}^{\prime}\right)}{D_{f}^{\prime}}
$$

where $\mathrm{m}^{\prime}$ is the perceived motion of the point of light associated with the sensed head motion $A^{\prime}, D_{n}^{\prime}$ is the perceived distance of the point of light from $O$, e.g., $D_{g}^{\prime}$ or $\mathrm{D}_{\mathrm{e}}^{\prime}$, and $\phi_{\mathrm{T}}^{\prime}$ is the sensed change in the direction of the point of light from $O$ expressed in radians. Thus, to find $m_{e}^{\prime}$ or $m_{g}^{\prime}, D_{e}^{\prime}$ or $D_{g}^{\prime}$ would be substituted for $D_{n}^{\prime}$ in Eq. 1. If $D_{f}^{\prime}$ and $A^{\prime}$ are known, $m^{\prime}$ can be predicted for any value of $D_{n}^{\prime}$. For the purposes of this study, it will be assumed, as a first approximation, that $\phi_{\mathrm{T}}^{\prime}=\phi_{\mathrm{T}}$ and $\mathrm{A}^{\prime}=\mathrm{A}$. In this case, Eq. 1 becomes

$$
m^{\prime}=\frac{A\left(D-D^{\prime}\right)}{D}
$$

where $D$ is the physical and $D^{\prime}$ the apparent distance of the point of light from the 0 . Equation 2 predicts that a point of light will appear to be stationary, or will appear to move in the direction of or opposite to the head motion, depending upon whether the perceived distance of the light is equal to, less than, or greater than its physical distance. The extent to which the assumptions $\phi_{\mathbf{T}}^{\prime}=\phi_{\mathbf{T}}$ and $\mathrm{A}^{\prime}=\mathrm{A}$ are justified must be determined in the particular case. It will be concluded that these assumptions are justified if it is found that direct measures of $D-D^{\prime}$, when applied to Eq. 2, produce predicted values of $\mathrm{m}^{\prime}$ that are in reasonable agreement with the values of $\mathrm{m}^{\prime}$ obtained from reports of the apparent motion. For this reason, in the present study, verbal reports were obtained of perceived distance as well as of perceived motion. Also, since the SDT is defined as the distance at which both $D-D^{\prime}$ and $m^{\prime}$ are zero, obtaining both $\mathrm{D}^{\prime}$ and $\mathrm{m}^{\prime}$ will permit a comparison of the value of the SDT computed from each of these measures.

Uncorrected verbal reports of perceived distance cannot be accepted as an unbiased measure of perceived distances. It has been found that when $O$ uses the memory of a foot ruler to indicate a perceived distance, he characteristically gives a response that is about .6 of the distance he actually perceives (Gogel, 1969). It is necessary, therefore, to calibrate the verbal reports of perceived distance in order to convert verbal reports to actual perceived distances. This calibration can be accomplished by having $\mathrm{O}$ report the apparent distance of objects located in a visual field containing many cues to distance (Gogel, 1968). From the assumption that distances are correctly perceived in the calibration field, a relation between verbal report and perceived distance (a calibration curve) can be determined and applied to the verbal reports of the distance of the point of light to convert the verbal reports to actual perceived distances.

It might also seem equally necessary to obtain calibration curves for verbal reports of displacement in order to convert reports of the magnitude of motion to perceived motion. But, in the present experiment, calibration is not as crucial for $\mathrm{m}^{\prime}$ as for $\mathrm{D}^{\prime}$. The reason for this is that in the determination of the SDT from $D^{\prime}$, an error involving a multiplicative constant in measuring $D^{\prime}$ will result in an error in the calculation of the SDT, i.e., when $D-D^{\prime}=0, D-K D^{\prime} \neq 0$, if $K \neq 1$. On the other hand, the specification of the SDT as the distance at which $\mathrm{m}^{\prime}=0$ will be unaffected by a multiplicative constant of calibration, i.e., when $\mathrm{m}^{\prime}=0, \mathrm{Km}^{\prime}=0$. Also, although a calibrated measure of $D-D^{\prime}$ will not be expected to predict an uncalibrated measure of $m^{\prime}$ using Eq. 2, the correlation of $D-D^{\prime}$ and $m^{\prime}$ as a function of $D$ obtained from a particular $O$ will not be affected by the lack of calibration of $\mathrm{m}^{\prime}$. Therefore, in the interest of restricting the amount of time required of 0 in the experiment, calibration corrections were obtained only for reports of perceived distance, not for reports of perceived magnitude of motion.

\section{METHOD}

\section{Observers}

The $O$ s were 60 students from an introductory course in psichology. All had at stereoasuity of at least $29 \mathrm{sec}$ of arc and a 
visual acuity of at least 20/30 near and far in both eyes as measured with a Keystone orthoscope.

\section{Apparatus}

Two observation positions were located in a lightproof booth. Each observation position faced an alley with independent lighting conditions in the two alleys. One of the observation positions and its alley was used for the experimental conditions and the other for the calibration condition.

\section{Experimental Conditions}

The observation position for the experimental conditions consisted of an adjustable head-and chinrest mounted on rollers so as to be movable laterally by $O$ through a distance of $13.5 \mathrm{~cm}$ from extreme left to extreme right. When binocular observation was used, the midpoint between the eyes moved $6.75 \mathrm{~cm}$ to the right and left of the straight-ahead direction to the points of light. When monocular observation was used (right eye only), the right eve moved $6.75 \mathrm{~cm}$ to the right and left of the straight-ahead direction to the points of light. Points of light were presented at distances of $30,91,183,457$. or $883 \mathrm{~cm}$ from $\mathrm{O}$. All points of light were presented one at a time along the same line of sight. perpendicular to the frontal plane of $O$, at the level of O's eyes, and were adjusted to appear to $E$ to be equally bright. The $O$ viewed the point of light through a $5 \times 22 \mathrm{~cm}$ aperture, which $E$ could close by a shutter when required. During the observations in the experimental conditions, neither the restrictive aperture nor any other object (or surface) was visible except the single point of light, i.e., the observation booth and the alley (except for the single point of light) were totally dark. Between presentations of the point of light at each of the five distances. the shutter was closed and a light in the observation booth was turned on. A microphone and earphones permitted $E$ and $O$ to communicate during the experiment. When required, clicks from a metronome were presented through the earphones at a rate of $1.6 / \mathrm{sec}$ to pace the right-left head movements. Between trials, white noise was presented in the earphones to mask any noise associated with the stimulus modifications for the next trial.

\section{Calibration Condition}

The observation position and the alley for the calibration condition were located to the right of the observation position and the alley for the experimental conditions. The floor of the calibration alley $(94.5 \mathrm{~cm}$ wide and $747 \mathrm{~cm}$ long, located $32 \mathrm{~cm}$ below the level of O's eyes) was covered with white cloth. The walls of the alley were formed by black curtains. White numbered squares $(10 \mathrm{~cm}$ on a side) were presented vertically on the alley floor at $38,114,200,394$, and $667 \mathrm{~cm}$ from $O$, with two of the squares presented on the left and three on the right of the midline of the floor. The alley in the calibration condition was illuminated by a series of overhead lights, with the floor and the walls as well as the numbered squares visible from the observation position. The observation position consisted of a stationary head- and chinrest. The observation in the calibration condition was always binocular.

\section{Procedure}

\section{Experimental Conditions}

The tasks of $O$ in the experimental conditions were explained with the use of a small model prior to entering the observation booth. The model was used to illustrate the situation in which a point of light could appear to be stationary despite head movement or could appear to move in the same or opposite direction as the head movement. Before being presented with any point of light. $O$ received practice in using the head-movement apparatus in the observation booth. The head movement always started from the extreme left or right position. It was explained that when the metronome clicks were presented. $O$ was to move the head- and chinrest by moving his head so that the head- and chinrest contacted a stop at either end of the movement simultaneously with the occurrence of a click. O was informed that, after completing four complete back-and-forth movements of his head for each presentation of a point of light. the metronome would be turned off and a shutter in front of the observation aperture would be closed. For each presentation of a point of light. $O$ had three tasks to be completed in the following order: (a) With head stationary. $O$ reported in feet or inches, or in some combination of feet and inches. the perceived distance of the point of light from his eves. (b) With the metronome turned on, $O$ moved his head in time with the metronome clicks while looking at the point of light. Following four head movements, the shutter was closed. and $O$ reported verbally whether the point of light had appeared stationary or moving and, if moving, whether it had appeared to move in the same direction as the head motion or opposite in direction to the head motion. To exclude reports of movement unrelated to head movement. only horizental motions of the point of light that changed direction with changes in direction of the head movement were to be reported. (c) If movement of the point of light was reported, $O$ was asked to indicate in feet or inches. or in some combination of feet and inches, the magnitude of the apparent right-to-left movement $\left(\mathrm{m}^{\prime}\right)$. All Os completed these tasks for each of the distances of the point of light (the experimental conditions) before being presented with the calibration condition. Thirty Os used binocular observation and 30 other Os used monocular observation throughout the experimental conditions. The $O s$ using monocular observation wore an opaque eve patch over their left eye.

\section{Calibration Condition}

Each $O$ indicated verbally in feet or inches, or in some combination of both, the distance that the numbered squares in the alley appeared to be from his eyes. All the numbered squares were present simultaneously, with each $O$ receiving a different random order for reporting the apparent distances of the squares.

\section{RESULTS}

Table 1 gives the number of $O s$ in the experimental conditions who saw the point of light at each distance as stationary (0), as moving in the same direction as the head movement $(+)$, or as moving in a direction opposite to the direction of the head movement (-) for both monocular and binocular observation. From the SDT, it was expected that the change in the perceived distance $D^{\prime}$ of the point of light would be less than the change in the physical distance $D$, with $D-D^{\prime}$ varying from a negative to a positive value as $D$ varied from a distance very close to $\mathrm{O}$ to a distance far from $\mathrm{O}$. Thus, from Eq. 2, the number of minus scores of perceived movement in Table 1 should decrease with increasing physical distance of the point of light and the number of positive scores should increase with increasing physical distance of the point of light from $O$. These expectations are in agreement with the pattern of results shown in Table 1. Clearly, the change in the direction of the perceived movement with increasing distance of the point of light from $O$ shown in Table 1 is consistent with 
Table 1

Frequencies With Which 30 Os Saw the Point of Light at Each Physical Distance as Moving Opposite to the Head Movement $(-)$, as Stationary $(0)$, or as Moving in the Direction of the Head Movement $(t)$

\begin{tabular}{lrrrrr}
\hline & \multicolumn{5}{c}{ Distance of Light (cm) } \\
\cline { 2 - 6 } & 30 & 91 & 183 & 457 & 883 \\
\hline & \multicolumn{5}{c}{ Monocular } \\
Opposite to Head (-) & 27 & 18 & 11 & 1 & 0 \\
Stationary (0) & 3 & 10 & 16 & 16 & 13 \\
Same as Head (+) & 0 & 2 & 3 & 13 & 17 \\
& \multicolumn{5}{c}{ Binocular } \\
Opposite to Head (-) & 17 & 13 & 8 & 6 & 4 \\
Stationary (0) & 13 & 15 & 16 & 19 & 12 \\
Same as Head (+) & 0 & 2 & 6 & 5 & 14 \\
\hline
\end{tabular}

Table 2

Verbal Reports of Distance, $D_{y}$ (Converted to Centimeters), Obtained from the Experimental and Calibration Conditions and the Results from Transforming the First by the Second into Perceived Distances, $D^{\prime}$

\begin{tabular}{|c|c|c|c|c|c|}
\hline & \multicolumn{5}{|c|}{$\begin{array}{c}\text { Calibration Conditions }(\mathrm{N}=60) \\
\text { Physical Distance }(\mathrm{cm})\end{array}$} \\
\hline & 38 & 113 & 200 & 394 & 667 \\
\hline Mean $D_{V}$ & 25 & 75 & 146 & 296 & 502 \\
\hline Median $D_{v}$ & 30 & 76 & 149 & 286 & 457 \\
\hline \multirow[t]{3}{*}{ SD of $D_{v}$} & 7 & 18 & 35 & 90 & 153 \\
\hline & \multicolumn{5}{|c|}{$\begin{array}{c}\text { Experimental Conditions }(\mathrm{N}=60) \\
\text { Physical Distance }(\mathrm{cm})\end{array}$} \\
\hline & 30 & 91 & 183 & 457 & 883 \\
\hline & \multicolumn{5}{|c|}{ Monocular Observation $(\mathrm{N}=30)$} \\
\hline Mean $D_{V}$ & 380 & 290 & 291 & 371 & 314 \\
\hline Median $D_{v}$ & 30 & 137 & 152 & 183 & 213 \\
\hline$S D$ of $D_{v}$ & 1130 & 392 & 430 & 435 & 314 \\
\hline Mean $\mathrm{D}^{\prime}$ & 489 & 410 & 422 & 488 & 425 \\
\hline Median $\mathrm{D}^{\prime}$ & 55 & 181 & 194 & 266 & 307 \\
\hline \multirow[t]{2}{*}{$\mathrm{SD}$ of $\mathrm{D}^{\prime}$} & 1667 & 677 & 651 & 661 & 466 \\
\hline & \multicolumn{5}{|c|}{ Binocular Observation $(N=30)$} \\
\hline Mean $\mathrm{D}_{\mathrm{v}}$ & 46 & 100 & 137 & 252 & 299 \\
\hline Median $D_{v}$ & 28 & 91 & 137 & 213 & 229 \\
\hline $\mathrm{SD}$ of $\mathrm{D}_{v}$ & 106 & 66 & 77 & 162 & 278 \\
\hline Mean $D^{\prime}$ & 55 & 128 & 159 & 282 & 309 \\
\hline Median $D^{\prime}$ & 34 & 102 & 158 & 256 & 276 \\
\hline $\mathrm{SD}$ of $\mathrm{D}^{\prime}$ & 93 & 89 & 64 & 152 & 196 \\
\hline
\end{tabular}

Table 3

Frequencies With Which for 30 Os the Physical Distance, D, of a Point of Light Was Less Than (Negative) or Greater Than (Positive) its Perceived Distance, $D^{\prime}$

\begin{tabular}{lllllrr}
\hline D of Light $(\mathrm{cm})$ & 30 & 91 & 183 & 457 & 883 \\
\hline & \multicolumn{5}{c}{ Monocular } \\
Negative & $\left(\mathrm{D}-\mathrm{D}^{\prime}\right)$ & 20 & 19 & 16 & 9 & 2 \\
Positive & $\left(\mathrm{D}-\mathrm{D}^{\prime}\right)$ & 10 & 11 & 14 & 21 & 28 \\
& & \multicolumn{5}{c}{ Binocular } \\
Negative & $\left(\mathrm{D}-\mathrm{D}^{\prime}\right)$ & 18 & 18 & 12 & 2 & 1 \\
Positive & $\left(\mathrm{D}-\mathrm{D}^{\prime}\right)$ & 12 & 12 & 18 & 28 & 29 \\
\hline
\end{tabular}

the results expected from the SDT and Eq. 2.

The verbal reports from the calibration condition were used to transform the verbal reports of distance obtained in the experimental conditions to perceived distances. The means, medians, and standard deviations of the reported distances in the experimental and calibration conditions (converted to centimeters) are shown in Table 2. The data from the calibration condition for each $\mathrm{O}$ were fitted by a power function using the linear line of best fit after transforming the physical distances and reported distances to logarithms. The calibration equation determined by the average coefficient and exponent from these individual power functions is $D_{v}=.64 D^{1.05}$, where $D_{v}$ is the reported and $D$ the physical distance of the numbered squares in the calibration condition. The individual calibration equations from the calibration condition were used to transform the verbal reports of distance obtained from each $O$ in the experimental conditions to perceived distance. The assumption involved in this transformation is that physical distances were veridically perceived in the full cue calibration conditions. Thus, the tendency for $\mathrm{O}$ to give verbal reports of distance that are about .6 of the physical distance (see the above power function) are assumed to reflect an error in the application of a foot ruler to the measurement of perceived distance, not an error in perceiving the distances of the squares in the calibration alley. It follows that if the verbal reports of distance, $D_{v}$, from the experimental conditions are substituted into the calibration equation for that $O$, the resulting values of $D^{\prime}$ obtained from this calibration equation are the actual perceived distances of the point of light (in the experimental condition) as indicated by the verbal reports. The means, medians, and standard deviations of the perceived distances, $D^{\prime}$, of the point of light obtained in this manner are also shown in Table 2. Since the $D_{v}$ and $D^{\prime}$ data are obviously skewed, medians, rather than means, are considered to best represent the results. The median $\mathrm{D}^{\prime}$ data indicate that for both monocular and binocular observation, the perceived distance of a point of light was less than its physical distance when the point of light was at a physical distance greater than about $2 \mathrm{~m}$ and, particularly in the case of monocular observation, was perceived to be greater than its physical distance when the physical distance was less than about $2 \mathrm{~m}$. These results are consistent with the postulation of an SDT.

From the perceived distances, $\mathrm{D}^{\prime}$, of the point of light, the number of times that the Os perceived the point of light as greater or less than its physical distance can be computed for each of the physical distances of the point of light. This is shown in Table 3 . It will be recalled from Eq. 2 that the apparent motion, $\mathrm{m}^{\prime}$. of the point of light should be negative (opposite to the head motion) when the perceived distance of the point of light is greater than its physical distance ( $D-D^{\prime}$ is -) 
and positive (in the same direction as the head motion) when the perceived distance of the point of light is less than its physical distance ( $D-D^{\prime}$ is + ). It will be seen from comparing Tables 1 and 3 that. in agreement with this prediction. both tables show an increase in the number of positive values and a decrease in the number of negative values with increasing physical distances of the point of light. The distance defined by the SDT can be computed independently from the frequency data of Table $1\left(\mathrm{~m}^{\prime}\right)$ and Table $3\left(\mathrm{D}-\mathrm{D}^{\prime}\right)$. According to Eq. 2. a point of light located at the distance of the SDT should appear stationary during head movement, since under these conditions $\mathbf{D}-\mathbf{D}^{\prime}=0$. Thus, the distance defined by the SDT can be calculated by determining the physical distance at which the + and - frequency curves of either Table 1 or Table 3 intersect. The results from the combined (monocular and binocular) frequency data are shown in Fig. 2. with the reports of no motion in Table 1 equally divided between the + and - data. The interpolated value of the SDT is $3.0 \mathrm{~m}$ from the perceived motion data and $1.5 \mathrm{~m}$ from the perceived distance data. Possibly the larger value of the SDT computed from the perceived motion data reflects a tendency, in agreement with a study by Hill (1972), for $\phi_{\mathrm{T}}^{\prime}$ to be somewhat less than $\phi_{\mathrm{T}}$. On the other hand, it is unlikely that this is the only factor involved, since $\phi_{T}^{\prime}$ would have to be about $1 / 2$ of $\phi_{\mathbf{T}}$ to account for a difference of this magnitude. A more likely explanation is that since $\phi_{T}$ decreases rapidly with distance (see Fig. 1), a very large difference in $D-D^{\prime}$ is required for large values of $D$ before motion is perceived, with the result that the measure of the SDT from the perceived motion data of Fig. 2 is inflated.

Figure 2 provides confirmation that the SDT occurs and that a discrepancy between perceived and physical distance can determine the direction of the perceived motion of a stationary object viewed while moving the head. It follows that the perceived motion of the stationary object resulting from head motion can provide a measure of the perceived distance of the object from $O$. This perceived distance is the physical distance at which no apparent movement occurs despite head movement. Such a measure of perceived distance might

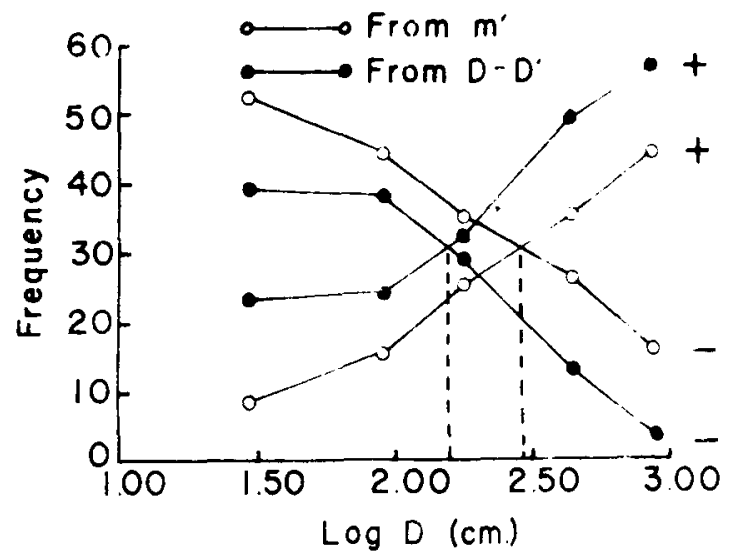

Fig. 2. Method of determining the value of the specific distance tendency from the perceived motion data $\left(m^{\prime}\right)$ and from the perceived distance data (D').

prove to be particularly useful in avoiding the response (cognitive) biases that sometimes occur with verbal reports of distance (Gogel \& Sturm, 1972).

It will be recalled that verbal reports of the magnitude as well as the direction of the perceived motion were obtained in the experimental conditions. Unfortunately. neither the mean nor the median values of $\mathrm{m}^{\prime}$ obtained as a function of the physical distance of the point of light can be considered to represent these data because of the rather large number of no-motion reports that occurred at several distances (see Table 1) and because of the occasional occurrence of very large reports of motion. To provide the reader with some indication of the order of magnitude of the reports of motion. however, Table 4 is presented. It will be noted in Table 4 that there was some tendency at the smallest value of $D$ for reports of motion to occur more frequently and to have a larger absolute magnitude for monocular as compared with binocular observation. This is consistent with the greater absolute magnitudes of $\mathrm{D}-\mathrm{D}^{\prime}$ obtained with this smallest value of $D$ for monocular as compared with binocular observation. as shown by the median data of Table 2.

Because of the problem in specifying a representative

Table 4

Frequency With Which Reports of Motion Occurred in Particular Intervals of Magnitude as a Function of the Physical Distance of the Point of Light

\begin{tabular}{|c|c|c|c|c|c|c|c|c|c|c|}
\hline \multirow[b]{2}{*}{ Interval $(\mathrm{cm})$} & \multicolumn{5}{|c|}{ 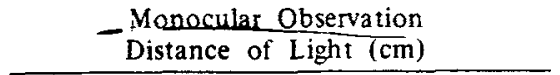 } & \multicolumn{5}{|c|}{$\begin{array}{l}\text { Binocular Observation } \\
\text { Distance of Light }(\mathrm{cm})\end{array}$} \\
\hline & 30 & 91 & 183 & 457 & 883 & 30 & 91 & 183 & 457 & 883 \\
\hline$<-15.2$ & 4 & 3 & 1 & 0 & 0 & 3 & 0 & 0 & 1 & 0 \\
\hline-15.2 to -7.6 & 12 & 3 & 1 & 0 & 0 & 3. & 4 & 1 & 0 & 0 \\
\hline-7.6 to 0.0 & 11 & 12 & 9 & 1 & 0 & 11 & 9 & 7 & 5 & 4 \\
\hline 0.0 & 3 & 10 & 16 & 16 & 13 & 13 & 15 & 16 & 19 & 12 \\
\hline 0.0 to +7.6 & 0 & 1 & 2 & 8 & 9 & 0 & 2 & 4 & 3 & 6 \\
\hline+7.6 to +15.2 & 0 & 0 & 0 & 4 & 5 & 0 & $\theta$ & 1 & 0 & 4 \\
\hline$>+15.2$ & 0 & 1 & 1 & 1 & 3 & 0 & 0 & 1 & 2 & 4 \\
\hline
\end{tabular}


Table 5

Distribution of Pearson Product-Moment Correlations Between $\mathbf{A}\left(\mathbf{D}-\mathbf{D}^{\prime}\right) / \mathrm{D}$ and $\mathrm{m}^{\prime}$ Obtained from Monocular and Binocular Observation

\begin{tabular}{|c|c|c|c|c|c|c|c|c|}
\hline Interval & $<.00$ & .00 to .25 & $\begin{array}{l}\text { Number of } \mathrm{r} \\
.25 \text { to } .50\end{array}$ & .50 to .75 & .75 to 1.00 & Mean $r$ & Median I & SD of $\mathrm{r}$ \\
\hline $\begin{array}{l}\text { Monocular } \\
\text { Binocular }\end{array}$ & $\begin{array}{l}4 \\
5\end{array}$ & $\begin{array}{l}1 \\
5\end{array}$ & $\begin{array}{l}4 \\
5\end{array}$ & $\begin{array}{l}4 \\
4\end{array}$ & $\begin{array}{l}17 \\
11\end{array}$ & $\begin{array}{l}.63 \\
.43\end{array}$ & $\begin{array}{l}.84 \\
.54\end{array}$ & $\begin{array}{l}.36 \\
.46\end{array}$ \\
\hline
\end{tabular}

Table 6

Average Rank of $A\left(D-D^{\prime}\right) / D$ and of $m^{\prime}$ as a Function of the Physical Distance, D, of the Point of Light

\begin{tabular}{lccccc}
\hline & \multicolumn{5}{c}{ D of Point $(\mathrm{cm})$} \\
\cline { 2 - 6 } & 30 & 91 & 183 & 457 & 883 \\
\hline \multirow{5}{*}{ Monocular } & 1.9 & 2.0 & 2.8 & 3.6 & 4.8 \\
Binocular & 1.8 & 1.7 & 2.8 & 3.8 & 4.9 \\
& & Average & Rank of & A(D $\left.-\mathrm{D}^{\prime}\right) / \mathrm{D}$ \\
Monocular & 1.3 & 2.3 & 3.0 & 4.1 & 4.2 \\
Binocular & 2.0 & 2.5 & 3.2 & 3.3 & 4.0 \\
\hline
\end{tabular}

measure of central tendency in the group data, individual rather than group data were used in the quantitative evaluation of Eq. 2. For this purpose, a Pearson product-moment correlation coefficient was computed between the $m^{\prime}$ and $A\left(D-D^{\prime}\right) / D$ data for each $O$ for the different values of $D$, with the $D^{\prime}$ being the corrected value of perceived distance obtained from the experimental conditions as calibrated from the data obtained in the calibration condition for that $O$. The distributions of obtained values of $r$ are indicated in Table 5. It will be noted that 26 of the obtained rs were positive with monocular observation and 25 were positive with binocular observation. The average value of $r$, also shown in Table 5, statistically was significantly different from zero beyond the .01 level (using a t test) for both monocular and binocular observation.

The $m^{\prime}$ and $A\left(D-D^{\prime}\right) / D$ data can be used to determine rank order data to further test Eq. 2. For this purpose, the $\mathrm{m}^{\prime}$ and $A\left(D-D^{\prime}\right) / D$ results for the

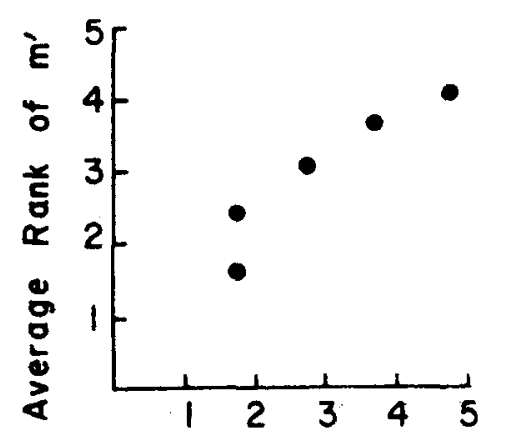

Average Rank of $A(D-D) / D$

Fig. 3. The relation between the perceived motion, $\mathbf{m}$ ', obtained directly and the perceived motion computed from perceived distance, $D^{\prime}$, using Eq. 2. different distances were ranked for each $O$, with the largest $A\left(D-D^{\prime}\right) / D$ result for that $O$ obtained at any of the five distances given the rank of 5 and the smallest given the rank of 1 . The smallest rank for either $\mathrm{m}^{\prime}$ or $A\left(D-D^{\prime}\right) / D$ for the $O$ was given to the smallest positive or largest negative value obtained at any of the five distances. The average ranks for $m^{\prime}$ and $A\left(D-D^{\prime}\right) / D$ as a function of $D$ are shown in Table 6 for both monocular and binocular observation. The $D^{\prime}$ values used to compute the $A\left(D-D^{\prime}\right) / D$ ranks of Table 6 are the verbal reports of distance as modified by the individual calibration equations. The plot of the rank order data combined for monocular and binocular observation is shown in Fig. 3. From Fig. 3, there is a clear tendency for the rank order data of $\mathrm{m}^{\prime}$ to be an increasing function of $A\left(D-D^{\prime}\right) / D$, as would be expected from Eq. 2. It is also likely, however, that Os differed in the degree to which $\phi_{\mathrm{T}}^{\prime}=\phi_{\mathbf{T}}$. This is indicated in that the Pearson product-moment correlation of $\mathrm{m}^{\prime}$ and $A\left(D-D^{\prime}\right) / D$ computed between Os at the same value of $D$ failed to be consistently positive. These values of $r$, in order of increasing $D$, are $-.11,-.49,+.28,+.18$, and -.13 for monocular observation and $+.89,-.25,-.14,+.07$, and -.29 for binocular observation.

\section{DISCUSSION}

The interpretation given to the results of the present study and the support from these results for this interpretation can be summarized as follows: In the absence of any cues to distance, a point of light will appear at a distance of about $2 \mathrm{~m}$ from $\mathrm{O}$ regardless of its physical distance. This is termed the specific distance tendency (SDT). If somewhat effective distance cues are present, such as the convergence and accommodation of the eyes, the light, although appearing at distances other than the distance defined by the SDT, will be displaced in apparent distance toward this distance. If the light is at a physical distance considerably beyond the $2 \mathrm{~m}$, to the degree that the convergence and accommodative cues are effective, the point of light will be perceived to be more distant than $2 \mathrm{~m}$. But, to the extent that convergence and accommodation are not completely effective in determining apparent distance, the effect of the SDT will be to make the light appear at a distance closer than that expected from the convergence and accommodative cues: Similarly, the SDT is expected to cause the apparent distance to be greater than that expected from the convergence or accommodative cue 
when the point of light is at a distance of less than about $2 \mathrm{~m}$. It follows that. under the rather reduced conditions of monocularly or binocularly viewing a point of light in a dark surround. D - D' will be positive for objects at a physical distance of greater than about $2 \mathrm{~m}$ and negative for objects at a distance of less than about $2 \mathrm{~m}$. From the research of Hay and Sawyer (1969) and Wallach, Yablick, and Smith (1972), it is expected that the perception of motion of the point of light with head movement will depend upon the apparent as well as the physical distance of the light from $O$. The expected effect of the difference between the apparent and physical distance of the light upon its apparent motion is described by Eq. 1 or, using the simplifying assumptions that $\phi_{\mathrm{T}}^{\prime}=\phi_{\mathrm{T}}$ and $\mathrm{A}^{\prime}=\mathrm{A}$, by Eq. 2 . In agreement with the SDT, it was found that both $\mathrm{D}-\mathrm{D}^{\prime}$ and $\mathrm{m}^{\prime}$ were negative for small values of $D$ and positive for large values of $D$. The greater magnitude of the SDT computed from the $\mathrm{m}^{\prime}$ as compared to the $\mathrm{D}-\mathrm{D}^{\prime}$ data was not interpreted as a valid difference. Some support for Eq. 2 is provided by the positive relation between the average ranks of $\mathrm{D}-\mathrm{D}^{\prime}$ and $\mathrm{m}^{\prime}$ and also by the generally positive correlations between $D-D^{\prime}$ and $m^{\prime}$ as a function of $D$. The lack of similar correlations between $D-D^{\prime}$ and $m^{\prime}$ for constant values of $D$ are thought to reflect $O$ differences in $\phi_{T}^{\prime}$ and $A^{\prime}$, which, if Eq. 1 is valid, suggests some limitations on the assumption that $\phi_{\mathrm{T}}^{\prime}=\phi_{\mathrm{T}}$ and $\mathrm{A}^{\prime}=\mathrm{A}$.

Although $O$ was informed that he was to look at the light while moving his head, fixation was not monitored during the experiment. It is possible, both in this experiment and in the previous experiments (Hay \& Sawyer, 1969: Wallach \& Frey, 1972; Wallach, Frey, \& Bode. 1972). that fixation was not always accurate, so that some retinal displacement of the light could have occurred during the head motion. In order to possibly explain the change from positive to negative perceived motion in the present study, however, this retinal motion would also have to change in direction as a function of physical distance. This would require an error in fixation distance that was less than the physical distance for the far distances of the light and greater than the physical distance for the near distances of the light and, in the present study, would need to be postulated for both monocular and binocular observation. 2 Such a fixation disparity has been identified for binocular fixation (Ogle, 1950). Whether or not it would occur for the successive fixation between spatially separated observation positions is unknown. But even if this latter type of fixation disparity were demonstrated as an appropriate function of physical distance, it would not necessarily follow that this fixation disparity would determine apparent motion. It is clear that retinal movement per se is neither a necessary nor a sufficient condition for perceived motion (see Mack, 1970). Furthermore, even if a successive fixation disparity had occurred and were important for the perceived motion. it would be concluded that at a distance of several meters. the monocular disparity approached zero (correct fixation). with this conclusion supporting the validity of the SDT. In addition, as will be discussed. Eq. $I$ can be applied to the condition in which two stationary objects are viewed simultaneously while moving the head. As will be reported in a manuscript in preparation. in this case. the difference between the physical and perceived distances of the objects is a significant factor in determining perceived relative motion as a result of head movement. Such a result cannot be explained in terms of any type of fixation errors occurring during the observations.

An alternative form of Eq. 1 that more clearly indicates the perceptual factors involved in the perception of motion of a physically stationary object with head motion is

$$
\mathrm{m}^{\prime}=\mathrm{A}^{\prime}-\phi_{\mathrm{T}}^{\prime} \mathrm{D}^{\prime}
$$

Equation 3 states that the perceived motion $\left(\mathrm{m}^{\prime}\right)$ is a function of the sensed (perceived) motion of the head. the change $\left(\phi_{T}^{\prime}\right)$ in the perceived direction of the object relative to $O$ at the terminal head positions, and the perceived distance $\left(\mathrm{D}^{\prime}\right)$ of the object. Applying Eq. 3 to the case in which two objects or points, "a" and "b." are presented simultaneously with head motion. it follows (since $A^{\prime}$ is the same for the two objects) that

$$
\mathrm{m}_{\mathrm{a}}^{\prime}-\mathrm{m}_{\mathrm{b}}^{\prime}=\phi_{\mathrm{T}_{\mathrm{b}}}^{\prime} \mathrm{D}_{\mathrm{b}}^{\prime}-\phi_{\mathrm{T}_{\mathrm{a}}}^{\prime} \mathrm{D}_{\mathrm{a}}^{\prime} \text {. }
$$

Equations 3 and 4 are useful in considering motion parallax as a cue to distance. The motion parallax cue to distance has been defined in two ways. In one definition, the important factor in the motion parallax cue to distance is considered to be the apparent movement of the objects (Helmholtz, 1925). According to this definition, $m^{\prime}$ in Eq. 3 and $m_{a}^{\prime}-m_{b}^{\prime}$ in Eq. 4 would be the absolute and relative cue of motion parallax, respectively. A second and more usual way of specifying motion parallax as a cue to distance is in terms of the change in direction of objects with respect to the moving head. In this case (see Graham, 1965), the cue of absolute motion parallax is the change in $\phi_{T}$, and the cue of relative motion parallax is the change in $\phi_{\mathbf{T}_{\mathbf{a}}}-\phi_{\mathbf{T}_{\mathbf{b}}}$.

There is evidence that relative motion parallax resulting from head motion can be an effective cue to the perception of depth between physically stationary objects and also some evidence that absolute motion parallax from head movement can provide a cue as to the distance of a physically stationary object from $O$ (Eriksson, 1972). But the results from the present study question the effectiveness of absolute motion parallax as a cue to distance defined in either of the above ways. As applied to the first definition. it is difficult to understand how in a particular observation perceived distance, as contained in the expression $D-D^{\prime}$. can simultaneousiy determine and be determined by 
perceived motion. In terms of the second definition, it should be noted that the change in the direction of perceived motion was in agreement with the concept of the SDT. This suggests that the error in perceived distance expected from the SDT occurred despite the presence of four back-and-forth movements of the head. At best, the SDT must be considered to be a relatively ineffective determiner of distance, since its effect is clear only in situations in which other distance cues are relatively reduced. In other words, since in the present experiment the SDT modified the reports of motion despite the presence of accommodation, convergence, and the motion parallax cue, neither of these cues can be regarded as having been particularly effective.

Although the results from the present study question the effectiveness of absolute motion parallax as a determiner of perceived egocentric distance, it should not be concluded that motion parallax is unimportant in distance perception. There is evidence that if $O$ had continued to move his head when presented with the point of light, $\mathrm{m}^{\prime}$ would soon have approached zero (Posin, 1966; Wallach \& Kravitz, 1965b; Wallach \& Frey, 1969; Wallach, Frey, \& Romney, 1969). It was for this reason that, in the present experiment, $O$ was limited to four head movements for each presentation of a point of light. This tendency for the perceived motion of a physically stationary point of light to decrease rapidly with continued head movement is an instance of perceptual learning. This perceptual learning could result either from a modification of $A^{\prime}, \phi_{T}^{\prime}$, or $D^{\prime}$ for constant values of $A, \phi_{T}$, and $D$, or from a change in the perception of motion associated with unchanged values of these perceptual variables. A study by Wallach, Yablick, and Smith (1972) suggests that much (but not all) of the perceptual learning associated with the perception of motion from head movement can be attributed to a recalibration of $D^{\prime}$, such that $D^{\prime}$ is modified in the direction of $\mathrm{D}$. Thus, although motion parallax from head movement may not be an immediately effective cue to distance, continued head movement possibly can result in the recalibration of perceived distances such that these perceived distances become increasingly veridical. Such rapid perceptual learning, if it occurs, can provide the important function of adjusting perceived space to be consistent with physical space. Rock (1966) has suggested a principle (the "concomitance principle") in terms of which such rapid perceptual learning might be explained. This principle states that $O$ discounts changes in retinal (or eye) position that are concomitant with sensed head movement. From the present point of view, it is suggested that perceived motion concomitant with sensed head motion provides information for the recalibration of perceived distance so that, in the case of physically stationary objects and continued head motion, perceived distance would become veridical and. in agreement with Eq. 2. perceived motion would approach zero. One consequence of this suggestion is that absolute motion parallax via this rapid perceptual learning would produce a veridical perception of egocentric distance which, in turn, could provide a veridical metric for perceptions of other extents in the visual field (Gogel, 1972).

The finding that a physically stationary object often appears stationary despite head movements (Wallach \& Kravitz, 1965) has been explained by a compensating process in which a central representation of the 0 's movement (efference) is compared with the changed stimulus input (reafference) resulting from the head movement (Gyr, 1972). But if $D-D^{\prime}$ is an important factor in determining $\mathrm{m}^{\prime}$, as this and the previous studies suggest, the comparison of efference and reafference information in determining the perception of motion must differ as a function of perceived distance. Furthermore, if the application of Eq. 3 to the situation in which several objects are presented simultaneously (Eq. 4) is valid, the efference-reafference compensation must be different for different portions of the visual field.

As was suggested, the perception of the direction of object motion when viewing a physically stationary object with the head moving can provide a measure of the perceived egocentric distance of the object with humans or animals. For this purpose, it will be assumed that $\phi_{\mathbf{T}}^{\prime}$ and $\mathrm{A}^{\prime}$ are essentially veridical. All that is required is to elicit some response indicating the direction of the apparent motion of the object for a given direction of head motion. The object can then be adjusted in distance (with constant cues to perceived distance) until the apparent motion disappears. At this adjusted distance, the perceived and physical distance are equivalent, i.e., the physical distance of the object will then be a measure of its apparent distance.

In summary, the results from the present study are interpreted as consistent with the following conclusions or implications: (1) The change in the direction of the apparent movement of the point of light relative to head movement provides evidence for the validity of the specific distance tendency (see Tables 1 and 3 and Fig. 2). (2) The direction and magnitude of the perceived motion of a stationary object as a result of head movement is a function of the apparent distance of the object from $O$ (see Tables 5 and 6 and Fig. 3). (3) The perception of object motion with head movement in the present study very likely represents a failure of the cue of motion parallax to determine perceived distance. It is suggested that the importance of head motion resides not so much in providing a cue to distance but rather in permitting, via perceptual learning, the rapid calibration of perceived space in the direction of making perceived spatial relations increasingly veridical. (4) The perception of object motion with a stationary object and moving head offers the possibility of an indirect method of measuring perceived egocentric distance. (5) The role of perceived distance in determining perceived movement as a result of head 
motion suggests that the relation between efference and reafference information in the perception of motion is not independent of perceived distance.

\section{REFERENCES}

Eriksson. E. S. Movement parallax and distance perception. Report 117. University of Uppsala. Uppsala. Sweden, 1972.

Gogel. W. C. The measurement of perceived size and distance. In W. D. Neff (Ed.). Contributions to senson' physiology. Vol. 3. New York: Academic Press. 1968.

Gogel. W. C. The sensing of retinal size. Vision Research, 1969, 9. 3-24.

Gogel. W. C. Scalar perceptions with binocular cues of distance. American Journal of Psychology. 1972, in press.

Gogel. W. C.. \& Sturm. R. D. Directional separation and the size cue to distance. Psychologische Forschung, 1971, 35. 57-80.

Graham. C. H. Visual space perception. In C. H. Graham (Ed.). l'ision and risual perception. New York: Wiley, 1965. Chap. 18.

Gyr. J. W. Is a theory of direct visual perception adequate? Psychological Bulletin. 1972, 77, 246-261.

Hay. J. C., \& Sawyer, S. Position constancy and binocular convergence. Perception \& Psychophysics, 1969, 5, 310-312.

Helmholtz. H. von. Heimholtz's treatise on physiological optics. (Translated and edited from the 3rd German ed. by J. P. C. Southall) Vol. 3. The perception of vision. The Optical Society of America, 1925. $295 \mathrm{pp}$.

Hill. A. L. Direction constancy. Perception \& Psychophysics, $1972,11.175-178$

Mack. A. An investigation of the relationship between eye and retinal image movement in the perception of movement. Perception \& Psychophysics, 1970, 8, 291-298.

Ogle, K. N. Researches in binocular vision. Philadelphia and London: Saunders, 1950.

Posin, R. L. Perceptual adaptation to contingent visual-field movement: An experimental investigation of position constancy. Doctoral dissertation, Yeshiva University. 1966.
(As discussed by I. Rock in The nature of perceptual adaptation. New York: Basic Books. 1966.)

Rock. 1. The nature of perceptual adaptation. New York: Basic Books, 1966.

Wallach. H.. \& Frey, K. J. Adaptation in the constancy of visual direction measured by a one-trial method. Perception \& Psychophysics, 1969. 5. 249-252.

Wallach. H. \& Frey, K. J. Adaptation in distance perception based on oculomotor cues. Perception \& Psychophysics. 1972. 11. 77-83.

Wallach. H., Frey, K. J.. \& Bode, K. A. The nature of adaptation in distance perception based on oculomotor cues. Perception \& Psychophysics, 1972, 11, 110-116.

Wallach. H., \& Kravitz, J. H. The measurement of the constancy of visual direction and of its adaptation. Psychonomic Science, 1965a. 2, 217-218.

Wallach, H., \& Kravitz, J. H. Rapid adaptation in the constancy of visual direction with active and passive rotation. Psychonomic Science, 1965b, 3. 165-166.

Wallach, H.. Yablick, G. S., \& Smith, A. Target distance and adaptation in distance perception in the constancy of visual direction. Perception \& Psychophysics. 1972. 11. 3-34.

\section{NOTES}

1. It has been demonstrated that the specific distance tendency can produce a difference between $D^{\prime}$ and $D$ for monocular observation of a point of light in an otherwise dark surround both when the physical distance of the point of light is greater than and when it is less than the distance of the specific distance tendency (Gogel, W. C. The effect of the specific distance tendency upon a monocularly observed object, in preparation). Also, it is likely that when the observation is binocular, some effect of the specific distance tendency will be evident, although this effect probably will be somewhat less than with monocular observation.

2. The authors wish to thank Whitman Richards for his suggestion of this possibility.

(Received for publication August 10, 1972; revision received November 17,1972 .) 